\title{
A técnica de clip-wrap: uma revisão de literatura
}

\author{
Daniella Brito Rodrigues ${ }^{1}$, Eberval Gadelha Figueiredo ${ }^{2}$, \\ Maria Luana Carvalho Viegas ${ }^{3}$, Saulo Araújo Teixeira ${ }^{4}$, Manoel Jacobsen Teixeira ${ }^{5}$
}

Divisão de Neurocirurgia da Faculdade de Medicina da Universidade de São Paulo (FMUSP), São Paulo, SP, Brasil.

\section{RESUMO}

A técnica de clip-wrap é utilizada quando o neurocirurgião é confrontado com aneurismas que não podem ser clipados pelas técnicas tradicionais, por causa de colo largo, calcificação, placas ateroscleróticas, aneurismas fusiformes ou quando há vasos oriundos do corpo do aneurisma. Diversos tipos de materiais podem ser utilizados, incluindo algodão, gaze de musselina, músculo, cola de fibrina e cianoacrilato. Nesta revisão de literatura, os autores destacam os resultados obtidos por esses diferentes materiais, assim como as complicações e a eficácia em relação às taxas de ressangramento e ruptura no pósoperatório. Conclui-se, portanto, que a técnica de clip-wrap é segura e eficaz, associada com uma baixa taxa de complicações pós-operatórias. O método pode impedir ressangramento e representa melhoria quando comparado com a história natural da doença.

\section{PALAVRAS-CHAVE}

Aneurisma intracraniano, aneurisma roto, procedimentos neurocirúrgicos.

\section{ABSTRACT}

The clip-wrap technique: a literature review

The clip-wrap technique is used when the neurosurgeon is confronted with aneurysms that cannot be cut by means of traditional techniques, due to a wide neck, calcification, atherosclerotic plaques, fusiforms aneurysms or when there are vessels from the aneurysm body. Various types of materials can be used, including cotton, gauze muslin, muscle, fibrin glue and cyanoacrylate. In this review, the authors highlight the results obtained by these different materials as well as the complications and efficacy in relation to the rates of rebleeding and rupture in the postoperative period. Therefore, the clip-wrap technique is safe and effective, associated with a low rate of postoperative complications. The method can prevent rebleeding and represents an improvement compared with the natural history of disease.

\section{KEYWORDS}

Intracranial aneurysm, aneurism ruptured, neurosurgical procedures.

Acadêmica de Medicina, Universidade do Estado do Pará (UEPA), Belém, PA, Brasil.

2 Supervisor da Divisão de Clínica Neurocirúrgica, chefe do Grupo de Neurocirurgia Vascular do Hospital das Clínicas da Universidade de São Paulo (USP), São Paulo, SP, Brasil.

3 Acadêmica de Medicina, Universidade Federal do Pará (UFPA), Belém, PA, Brasil.

4 Acadêmico de Medicina, Universidade Federal do Ceará (UFC), Fortaleza, CE, Brasil.

5 Diretor da Divisão de Clínica Neurocirúrgica do Hospital das Clínicas, professor titular da Disciplina de Neurocirurgia da USP, São Paulo, SP, Brasil. 


\section{Introdução}

Quando um aneurisma cerebral não é acessível à clipagem cirúrgica ou ao tratamento endovascular, a técnica de clip-wrap é uma alternativa para reduzir o risco de ruptura e ressangramento. ${ }^{1-4}$ Esse método é geralmente utilizado quando há lesões que não podem ser clipadas ou apenas parcialmente clipadas, por causa de colo alargado, calcificação, placas ateroscleróticas, formato fusiforme ou quando há vasos oriundos do corpo do aneurisma. ${ }^{2}$ Primeiramente realizada por Dott, ${ }^{1}$ em 1933, que tratou um aneurisma de artéria cerebral média revestindo-o com músculo, a técnica de clip-wrap consiste no reforço da parede de aneurismas não clipáveis ou apenas parcialmente clipáveis, por meio do revestimento (wrapping) com materiais como algodão, músculo, gaze de musselina, surgicel e cianoacrilato.

Apesar de a clipagem do colo do aneurisma ser, geralmente, considerada o tratamento de escolha para os aneurismas intracerebrais, ${ }^{5-7}$ muitos autores relataram a eficácia do clip-wrap, assim como as pequenas taxas de ressangramento nas lesões tratadas por essa técnica. ${ }^{3,5,8-12}$ O presente estudo tem como objetivo realizar uma revisão de literatura acerca das principais indicações, materiais e complicações e da eficácia da técnica de clip-wrap como opção terapêutica em aneurismas não clipáveis pelas técnicas convencionais.

\section{Indicações}

A literatura descreve como principais indicações da técnica de clip-wrap os casos em que não é possível preservar os ramos perfurantes das artérias com o clip, aneurismas de colo largo, aneurismas fusiformes, calcificação, ruptura pré-operatória do colo aneurismático ou quando há algum ateroma impedindo a clipagem., ${ }^{2,3,12-14}$

\section{Materiais e complicações}

No primeiro relato de wrapping, Dott ${ }^{1}$ utilizou músculo para envolver um aneurisma da artéria cerebral média e, desde então, diversos materiais têm sido utilizados com esse propósito. Anos depois, em 2008, Suh et al. ${ }^{12}$ voltaram a analisar a eficácia do músculo temporal como envoltório de aneurismas não clipáveis pelas técnicas tradicionais e obtiveram resultados favoráveis em relação às taxas de ressangramento e ruptura, embora outros autores tenham observado complicações hemorrágicas no pós-operatório após uso de músculo. ${ }^{2,6}$
Bederson et al. ${ }^{8}$ obtiveram bons resultados ao tratar aneurismas fusiformes e ateroscleróticos reforçando a parede aneurismática por meio de gaze de musselina com cortes adaptáveis ao formato do aneurisma e suas artérias perfurantes, objetivando a preservação de tais ramos, e com posterior clipagem circunferencial. Apesar dos resultados favoráveis encontrados por esses autores, complicações infecciosas relacionadas com a musselina são bem descritas na literatura, ${ }^{15,16}$ principalmente a aracnoidite óptica, ou "gauzoma", que evolui com perda visual, documentada por autores como Fujitsu et al. ${ }^{14}$ Felsberg et al. ${ }^{17}$ McFadzean et al. ${ }^{18}$ e Prabhu et al. ${ }^{19}$ que também relataram técnicas de wrapping com o intuito de preservar os ramos perfurantes das artérias e descreveram uma técnica usando silastic revestido com malha de Dacron, com formato similarmente adaptável para preservar nervos cranianos e ramificações, e posterior clipagem circunferencial sobre o silastic.

Outros autores descreveram a criação de um envoltório para aneurismas de Gore-Tex com o mesmo princípio da flexibilidade e melhor preservação de estruturas adjacentes. ${ }^{13,20}$ Ainda sobre a utilização de Gore-Tex, Kim et al. ${ }^{20}$ trataram aneurismas fusiformes enovelando Gore-Tex em um clip adaptado e concluíram que esse método proporciona maior segurança à parede aneurismática, bem como menor reação tecidual.

Herrera et al. ${ }^{21}$ realizaram o primeiro estudo histológico em ratos, testando três materiais: cola de fibrina, cola de cianoacrilato e tecido de algodão; esses autores concluíram que a cola de cianoacrilato (Biobond) induziu alterações inflamatórias crônicas, necrose e estreitamento luminal por fibrose, tanto na túnica média quanto na adventícia, em um modelo arterial de cobaias. Os resultados obtidos sugerem que a cola de cianoacrilato pode causar as lesões arteriais oclusivas observadas após a cirurgia. Similarmente, Minakawa et al. ${ }^{10}$ relataram elevada taxa de ressangramento após a utilização do Biobond. Alguns materiais de wrapping foram estudados em laboratório por autores como Sadasivan et al. ${ }^{22} \mathrm{e}$ Sachs, ${ }^{23}$ que encontraram que músculo e colágeno utilizados são absorvidos e não induzem reação fibrótica, enquanto Ebina et al. ${ }^{7}$ descobriram que músculo, fáscia e dura-máter se tornaram necróticos na parede aneurismática.

Deshmukh et al., ${ }^{3}$ ao analisarem a evolução em longo prazo de uma série de casos de aneurismas não clipáveis pelas técnicas convencionais, sugeriram que o uso do algodão pode ser preferível em relação ao Biobond e à cola de fibrina, pois constataram que o algodão tem resposta inflamatória menos significativa. Esses autores recomendam, portanto, o uso de algodão para o revestimento dos aneurismas, pela simplicidade e menor reação fibrótica. Kubo et al. ${ }^{24}$ relataram clip-wrap com politetrafluoretileno (PTFE) em aneurismas da artéria carótida interna (ACI) em seis pacientes. Não 
houve ressangramentos ou outras complicações no pós-operatório; os autores concluíram, portanto, que o wrap-clipping com PTFE é um procedimento útil para a gestão dos aneurismas rotos da $\mathrm{ACI}$, além de ter resistência suficiente, baixa reatividade e elevada biocompatibilidade, tornando-o seguro para o implante nos seres humanos. ${ }^{14,25-27}$

Em 2010, Figueiredo et al. ${ }^{28}$ analisaram a casuística de nove casos de pacientes tratados pela técnica de clip-wrap e destacaram a preferência pelo Rayon (seda artificial) para revestimento dos aneurismas, o qual assegura que certa tensão seja exercida sobre a parede arterial e, em seguida, pode ser aplicada também uma cola biológica para promover maior firmeza. Esses autores destacaram como complicação mais frequente os granulomas tardios na região do wrapping, que podem provocar neuropatias cranianas, desse modo o Rayon não deve ser colocado em contato direto com os nervos cranianos.

\section{Eficácia}

$\mathrm{Na}$ era pré-microscópica da neurocirurgia, o ato de revestir ou wrapping os aneurismas apresentava risco significativamente maior de complicação intraoperatória ou ressangramento. Com o desenvolvimento de equipamentos e de técnicas microcirúrgicas, a melhora na exposição do colo do aneurisma teve como consequência menor risco de lesões em vasos e tecidos adjacentes, tornando, desse modo, a técnica de clip-wrap mais segura e eficaz. ${ }^{7}$

A literatura relata que a taxa de ressangramento de aneurismas tratados pela técnica de clip-wrap é geralmente baixa, ${ }^{3,6,29}$ no entanto não tem a mesma eficácia da clipagem tradicional. Todd et al. ${ }^{6}$ relataram taxa de ressangramento nos primeiros seis meses de $8,6 \%$, enquanto a de ressangramento tardio foi de $1,5 \%$ por ano. Para Suh et al. ${ }^{12}$, em 2008, seus resultados com o clip-wrap tiveram uma taxa de segurança mais elevada em relação aos demais estudos; eles concluíram que o uso concomitante do clip com o invólucro é ainda mais eficaz do que a técnica de revestimento simples. Tais estudos são concordantes com o de Figueiredo et al. , $^{28}$ que, em 2010, relatou ausência de ressangramento precoce e tardio em todos os seus pacientes analisados e concluiu, portanto, que o uso do clip-wrap representa um avanço em relação à história natural dos aneurismas não clipáveis.

\section{Considerações finais}

A literatura especializada considera a técnica de clip-wrap segura e eficaz, associada com baixa taxa de complicações pós-operatórias, apesar de não ser tão efetiva quanto a clipagem tradicional. Ela pode impedir ressangramento e representa melhoria quando comparada com a história natural da doença.

\section{Referências}

1. Dott NM. Intracranial aneurysms: cerebral arterioradiography: surgical treatment. Edinb Med J. 1933;40:21934.

2. Cossu M, Pau A, Turtas S, Viola C, Viale GL. Subsequent bleeding from ruptured intracranial aneurysms treated by wrapping or coating: a review of the long-term results in 47 cases. Neurosurgery. 1993;32(3):344-6.

3. Deshmukh VR, Kakarla UK, Figueiredo EG, Zabramski JM, Spetzler RF. Long-term clinical and angiographic follow-up of unclippable wrapped intracranial aneurysms. Neurosurgery. 2006;58(3):434-42.

4. Mount LA, Antunes JL. Results of treatment of intracranial aneurysms by wrapping and coating. J Neurosurg. 1975;42(2):189-93.

5. Todd NV, Howie JE, Miller JD. Norman Dott's contribution to aneurysm surgery. J Neurol Neurosurg Psychiatry. 1990;53(6):455-8.

6. Todd NV, Tocher JL, Jones PA, Miller JD. Outcome following aneurysm wrapping: a 10-year follow-up review of clipped and wrapped aneurysms. J Neurosurg. 1989;70(6):841-6.

7. Ebina K, Iwabuchi T, Suzuki S. A clinico-experimental study on various wrapping materials of cerebral aneurysms. Acta Neurochir (Wien). 1984;72(1-2):61-71.

8. Bederson JB, Zabramski JM, Spetzler RF. Treatment of fusiform intracranial aneurysms by circumferential wrapping with clip reinforcement. Technical note. J Neurosurg. 1992;77(3):478-80.

9. Fujiwara S, Fujii K, Nishio S, Fukui M. Long-term results of wrapping of intracranial ruptured aneurysms. Acta Neurochir (Wien). 1990;103(1-2):27-9.

10. Minakawa T, Koike T, Fujii Y, Ishii R, Tanaka R, Arai H. Long term results of ruptured aneurysms treated by coating. Neurosurgery. 1987;21(5):660-3.

11. Sato K, Fujiwara S, Kameyama M, Ogawa A, Yoshimoto T, Suzuki J. Follow-up study on ruptured aneurysms treated by wrapping. Neurol Med Chir (Tokyo). 1990;30(10):734-7.

12. Suh SJ, Kim SC, Kang DG, Ryu KY, Lee HG, Cho JH. Clinical and angiographic results after treatment with combined clipping and wrapping technique for intracranial aneurysm. J Korean Neurosurg Soc. 2008;44(4):190-5.

13. Kato $\mathrm{Y}$, Sano H, Kanno T. Creation of an instant wrapping clip with GORE-TEX ${ }^{\circledR}$ sheet. J Clin Neuroscience. 1997;4(4):486-7.

14. Fujitsu K, Ishiwata Y, Gondo G, Fujii S, Feng DD. Wrapclipping with a Dacron mesh silastic sheet. Technical note. J Neurosurg. 1994;80(2):336-7.

15. Andres RH, Guzman R, Weis J, Schroth G, Barth A. Granuloma formation and occlusion of an unruptured aneurysm after wrapping. Acta Neurochir (Wien). 2007;149(9):953-8.

16. Cudlip SA, Kitchen ND, McKhahn GM, Bell BA. Wrapping of solitary ruptured intracranial aneurysms, outcome at five years. Acta Neurochir (Wien). 1998;140(11):1167-70. 
17. Felsberg GJ, Tien RD, Haplea S, Osumi AK. Muslin-induced optic arachnoiditis ("gauzoma"): findings on CT and MR. J Comput Assist Tomogr. 1993;17(3):485-7.

18. McFadzean RM, Hadley DM, Mcllwaine GG. Optochiasmal arachnoiditis following muslin wrapping of ruptured anterior communicating artery aneurysms. J Neurosurg. 1991;75(3):393-6.

19. Prabhu SS, Keogh AJ, Parekh HC, Perera S. Optochiasmal arachnoiditis induced by muslin wrapping of intracranial aneurysms. A report of two cases and a review of the literature. Br J Neurosurg. 1994;8(4):471-6.

20. Kim LJ, Klopfenstein JD, Spetzler RF. Clip reconstruction and sling wrapping of a fusiform aneurysm: technical note. Neurosurgery. 2007;61(Suppl 3):79-80.

21. Herrera O, Kawamura S, Yasui N, Yoshida Y. Histological changes in the rat common carotid artery induced by aneurysmal wrapping and coating materials. Neurol Med Chir (Tokyo). 1999;39(2):134-9.

22. Sadasivan B, Ma S, Dujovny M, Ho LK, Ausman JI. Use of experimental aneurysms to evaluate wrapping materials. Surg Neurol. 1990;34(1):3-7.

23. Sachs $E$ Jr. The fate of muscle and cotton wrapped about intracranial carotid arteries and aneurysms. A laboratory and clinico-pathological study. Acta Neurochir (Wien). 1972;26(2):121-37.

24. Kubo Y, Ogasawara K, Tomitsuka N, Otawara Y, Watanabe M, Ogawa A. Wrap-clipping with polytetrafluoroethylene for ruptured blisterlike aneurysms of the internal carotid artery. Technical note. J Neurosurg. 2006;105(5):785-7.

25. Aliredjo RP, de Vries J, Menovsky T, Grotenhuis JA, Merx $J$. The use of Gore-Tex membrane for adhesion prevention in tethered spinal cord surgery: technical case reports. Neurosurgery. 1999;44(3):674-7.

26. Nakagawa S, Hayashi T, Anegawa S, Nakashima S, Shimokawa S, Furukawa Y. Postoperative infection after duraplasty with expanded polytetrafluoroethylene sheet. Neurol Med Chir (Tokyo). 2003;43(3):120-4.

27. Shigeta H, Kyoshima K, Nakagawa F, Kobayashi S. Dorsal internal carotid artery aneurysms with special reference to angiographic presentation and surgical management. Acta Neurochir (Wien). 1992;119(1-4):42-8.

28. Figueiredo EG, Foroni L, Monaco BA, Gomes MQ, Sterman Neto H, Teixeira MJ. The clip-wrap technique in the treatment of intracranial unclippable aneurysms. Arq Neuropsiquiatr. 2010;68(1):115-8.

29. Taylor JC, Choudhury AR. Reinforcement with gauze wrapping for ruptured aneurysms of the middle cerebral artery. J Neurosurg. 1977;47(6):828-32.

\section{Endereço para correspondência}

Daniella Brito Rodrigues

Rua Silva Castro, 559, Guamá

66075-104 - Belém, PA, Brasil

Telefone: (91) 8815-7806

E-mail: britodaniella32@gmail.com 